PROCEEDINGS OF THE

AMERICAN MATHEMATICAL SOCIETY

Volume 126, Number 7, July 1998, Pages 2033-2037

S $0002-9939(98) 04438-4$

\title{
HIGHER ORDER TURÁN INEQUALITIES
}

\author{
DIMITAR K. DIMITROV
}

(Communicated by J. Marshall Ash)

\begin{abstract}
The celebrated Turán inequalities $P_{n}^{2}(x)-P_{n-1}(x) P_{n+1}(x) \geq$ $0, \quad x \in[-1,1], \quad n \geq 1$, where $P_{n}(x)$ denotes the Legendre polynomial of degree $n$, are extended to inequalities for sums of products of four classical orthogonal polynomials. The proof is based on an extension of the inequalities $\gamma_{n}^{2}-\gamma_{n-1} \gamma_{n+1} \geq 0, \quad n \geq 1$, which hold for the Maclaurin coefficients of the real entire function $\psi$ in the Laguerre-Pólya class, $\psi(x)=\sum_{n=0}^{\infty} \gamma_{n} x^{n} / n$ !
\end{abstract}

\section{INTRODUCTION AND STATEMENT OF RESULTS}

For any sequence of polynomials $\left\{p_{n}\right\}_{n=0}^{\infty}$ the quantities $\Delta_{n}(p ; x):=p_{n}^{2}(x)-$ $p_{n-1}(x) p_{n+1}(x)$ are called Turán determinants, associated with $\left\{p_{n}\right\}_{n=0}^{\infty}$. Szegö [18] was the first to call attention to the following beautiful inequalities of P. Turán:

$$
\Delta_{n}(P ; x)=P_{n}^{2}(x)-P_{n-1}(x) P_{n+1}(x) \geq 0, \quad x \in[-1,1], \quad n \geq 1 .
$$

In the same paper Szegö obtained extensions of (1) to Gegenbauer (ultraspherical), Laguerre and Hermite polynomials. Karlin and Szegö [10] proved that certain higher order Turán determinants for the same classes of classical orthogonal polynomials do not change their sign in the interval of orthogonality. Gasper [9] proved the analog of (1) for a class of Jacobi polynomials. Askey's comments on [10] and [18] in Volume 3 of Szegö's collected papers survey further contributions and developments.

The reason for the recent interest in Turán determinants is that for the orthogonal polynomials $\left\{p_{n}\right\}_{n=0}^{\infty}$ in a subclass of the class $M(0,1)$ the quantities $\Delta_{n}(p ; x)$ converge uniformly on the compact subsets of $(-1,1)$ to $2\left(1-x^{2}\right)^{1 / 2} /\left(\pi \alpha^{\prime}(x)\right)$, where $\alpha^{\prime}(x)$ is the absolutely continuous part of the measure, with respect to which the $p_{n}$ are orthogonal $[5,6,7,12,19]$.

Szegó [18] gives Turan's proof and three additional proofs of (1). The third proof is particularly ingenious and allows the extension of (1) to the ultraspherical, Laguerre and Hermite polynomials. Szegő atributes the idea of this proof to Pólya.

Received by the editors December 12, 1996.

1991 Mathematics Subject Classification. Primary 30D10, 33C45.

Key words and phrases. Turán inequalities, Turán determinants, entire functions in the Laguerre-Pólya class, Riemann hypothesis.

Research supported by the Brazilian foundation CNPq under Grant 300645/95-3 and the Bulgarian Science Foundation under Grant MM-414.

(C)1998 American Mathematical Society 
A real entire function

$$
\psi(x)=\sum_{n=0}^{\infty} \gamma_{n} \frac{x^{n}}{n !}
$$

is said to belong to the Laguerre-Pólya class $(\psi \in \mathcal{L}-\mathcal{P})$ if

$$
\psi(x)=c x^{m} e^{-\alpha x^{2}+\beta x} \prod_{k=1}^{\infty}\left(1+x / x_{k}\right) e^{-x / x_{k}},
$$

where $c, \beta, x_{k}$ are real, $\alpha \geq 0, m$ is a nonnegative integer and $\sum x_{k}^{-2}<\infty$. We have adopted the notations in $[2,4,14]$, which one may consult for the important properties of the functions in Laguerre-Pólya class. Generally, $\mathcal{L}-\mathcal{P}$ consists of entire functions which are uniform limits on the compact sets of the complex plane of real polynomials with only real zeros. A necessary condition that $\psi \in \mathcal{L}$ - $\mathcal{P}$ is that its Maclaurin coefficients satisfy (cf. $[2,4,16])$

$$
\gamma_{n}^{2}-\gamma_{n-1} \gamma_{n+1} \geq 0, \quad n \geq 1
$$

Then, in order to prove the inequalities

$$
\Delta_{n}(p ; x) \geq 0, \quad n \geq 1
$$

where
a) $p_{n}(x)=P_{n}^{(\lambda)}(x) / P_{n}^{(\lambda)}(1) \quad$ for $\quad x \in[-1,1], \quad \lambda>-1 / 2$,

b) $p_{n}(x)=L_{n}^{(\alpha)}(x) / L_{n}^{(\alpha)}(0)$ for $x \in[0, \infty), \quad \alpha>-1$,

or

c) $p_{n}(x)=H_{n}(x)$ for $x \in(-\infty, \infty)$,

where $P_{n}^{(\lambda)}, L_{n}^{(\alpha)}$ and $H_{n}$ denote the ultraspherical, Laguerre and Hermite polynomials, one uses (3) together with the fact that the generating functions which appear on the right-hand sides of

$$
\begin{gathered}
\sum_{n=0}^{\infty} \frac{P_{n}^{(\lambda)}(x)}{P_{n}^{(\lambda)}(1)} \frac{z^{n}}{n !}=2^{\lambda-1 / 2} \Gamma(\lambda+1 / 2) e^{x z} \frac{J_{\lambda-1 / 2}\left(\left(1-x^{2}\right)^{1 / 2} z\right)}{\left(\left(1-x^{2}\right)^{1 / 2} z\right)^{\lambda-1 / 2}}, \quad \lambda>-1 / 2, \\
\sum_{n=0}^{\infty} \frac{L_{n}^{(\alpha)}(x)}{L_{n}^{(\alpha)}(0)} \frac{z^{n}}{n !}=\Gamma(\alpha+1) e^{z} \frac{J_{\alpha}\left(2(x z)^{1 / 2}\right)}{(x z)^{\alpha / 2}}, \quad \alpha>-1,
\end{gathered}
$$

and

$$
\sum_{n=0}^{\infty} H_{n}(x) \frac{z^{n}}{n !}=e^{2 x z-z^{2}}
$$

are in the Laguerre-Pólya class.

Another reason that inequalities (3) are interesting is their connection to the celebrated Riemann hypothesis [17] about the zeros of the Riemann $\zeta$-function. It is well known and easy to see that the Riemann hypothesis holds true if and only if the Riemann $\xi$-function, defined by

$$
\xi(i z)=\frac{1}{2}\left(z^{2}-1 / 4\right) \pi^{-z / 2-1 / 4} \Gamma(z / 2+1 / 4) \zeta(z+1 / 2),
$$

has only real zeros. It is known that $\xi$ is a real entire function of order one. It can be represented in the form

$$
\xi(x / 2)=8 \int_{0}^{\infty} \Phi(t) \cos x t d t,
$$


where

$$
\Phi(t)=\sum_{n=1}^{\infty}\left(2 n^{4} \pi^{2} e^{9 t}-3 n^{2} \pi e^{5 t}\right) \exp \left(-n^{2} \pi e^{4 t}\right)
$$

Then

$$
\frac{1}{8} \xi(x / 2)=\sum_{k=0}^{\infty}(-1)^{k} \hat{b}_{k} \frac{x^{2 k}}{(2 k) !}
$$

with

$$
\hat{b}_{k}=\int_{0}^{\infty} t^{2 k} \Phi(t) d t, \quad \text { for } \quad k \geq 0 .
$$

On setting $z=-x^{2}$ we obtain the entire function

$$
\xi_{1}(z)=\sum_{k=0}^{\infty} \hat{\gamma}_{k} \frac{z^{k}}{k !}, \quad \hat{\gamma}_{k}=\frac{k !}{(2 k) !} \hat{b}_{k},
$$

of order $1 / 2$. Therefore, by the Hadamard theorem, the Riemann hypothesis is equivalent to the statement that $\xi_{1} \in \mathcal{L}-\mathcal{P}$ (cf. Pólya and Schur [16] and Boas [1, p. 24]). Hence the inequalities $\hat{\gamma}_{n}^{2}-\hat{\gamma}_{n-1} \hat{\gamma}_{n+1} \geq 0, n \geq 1$, which are equivalent to the inequalities $(2 n+1) \hat{b}_{n}^{2}-(2 n-1) \hat{b}_{n-1} \hat{b}_{n+1} \geq 0, n \geq 1$, are necessary conditions for the Riemann hypothesis to be true. Craven, Norfolk and Varga [3] proved the latter inequalities, thus verifying a conjecture of Pólya [15] (see also Varga [20, Chapter 3]).

In this paper we obtain, in a very simple way, new necessary conditions for a real entire function to belong to $\mathcal{L}-\mathcal{P}$. These condition are extensions of $(3)$. Then the idea of Pólya, sketched above, immediately yields extensions of (1).

Theorem 1. Let the real entire function $\psi$, defined by (2), be in the LaguerrePólya class. Then

$$
4\left(\gamma_{n}^{2}-\gamma_{n-1} \gamma_{n+1}\right)\left(\gamma_{n+1}^{2}-\gamma_{n} \gamma_{n+2}\right)-\left(\gamma_{n} \gamma_{n+1}-\gamma_{n-1} \gamma_{n+2}\right)^{2} \geq 0 \quad \text { for } \quad n \geq 1 .
$$

Corollary 1. Let $\hat{\gamma}_{n}$ be defined by (4), (5) and (6). A necessary condition that the Riemann hypothesis holds true is that the inequalities

$$
4\left(\hat{\gamma}_{n}^{2}-\hat{\gamma}_{n-1} \hat{\gamma}_{n+1}\right)\left(\hat{\gamma}_{n+1}^{2}-\hat{\gamma}_{n} \hat{\gamma}_{n+2}\right)-\left(\hat{\gamma}_{n} \hat{\gamma}_{n+1}-\hat{\gamma}_{n-1} \hat{\gamma}_{n+2}\right)^{2} \geq 0, \quad n \geq 1,
$$

hold.

Corollary 2. The inequalities

$$
\begin{aligned}
\delta_{n}(p ; x):= & 4\left(p_{n}^{2}(x)-p_{n-1}(x) p_{n+1}(x)\right)\left(p_{n+1}^{2}(x)-p_{n}(x) p_{n+2}(x)\right) & \\
& -\left(p_{n}(x) p_{n+1}(x)-p_{n-1}(x) p_{n+2}(x)\right)^{2} \geq 0, & n \geq 1,
\end{aligned}
$$

hold for the classes of orthogonal polynomials a), b) and c), described above.

\section{Proof OF THE THEOREM AND REMARKS}

Proof of the theorem. Let the real entire function $\psi$, defined by (2), be in the Laguerre-Pólya class. Then, for any positive integer $n$, the $n$-th associated Jensen polynomial

$$
g_{n}(x):=\sum_{k=0}^{n}\left(\begin{array}{l}
n \\
k
\end{array}\right) \gamma_{k} x^{k}
$$


has only real zeros (cf. $[2,4,15])$. Observe that for any $q \leq n$

$$
g_{n}^{(q)}(x)=\frac{n !}{(n-q) !} g_{n-q, q}(x),
$$

where

$$
g_{n, q}(x):=\sum_{k=0}^{n}\left(\begin{array}{c}
n \\
k
\end{array}\right) \gamma_{k+q} x^{k}, \quad n=0,1, \ldots,
$$

are the Jensen polynomials associated with $\psi^{(q)}$. Then Rolle's theorem implies that for any positive integer $n$ and any nonnegative integer $q$ the polynomial $g_{n, q}(x)$ has only real zeros. The latter follows also from the fact that the class $\mathcal{L}-\mathcal{P}$ is closed under differentiation (cf. Pólya and Schur [16]). Now the assertion of the theorem follows from a result of Mař́k [11] (see also [13, Theorem 1.3.3 on p. 99]). It states that if the real polynomial

$$
p(x)=\sum_{k=0}^{n} a_{k} x^{k} /(k !(n-k) !)
$$

of degree $n \geq 3$ has only real zeros, then the inequalities

$$
4\left(a_{k}^{2}-a_{k-1} a_{k+1}\right)\left(a_{k+1}^{2}-a_{k} a_{k+2}\right)-\left(a_{k} a_{k+1}-a_{k-1} a_{k+2}\right)^{2} \geq 0, \quad 1 \leq k \leq n-2,
$$

hold.

Corollary 1 is immediate. In order to prove Corollary 2 one uses the statement of Theorem 1 and the idea of Pólya, described in the first section.

A natural conjecture is that inequalities (7) hold true. Numerical calculations, based on the values of the first twenty coefficients $\hat{b}_{n}$, given in [3], support the conjecture.

It is interesting to see what is the limit of the quantities $\delta_{n}(p ; x)$ for the class of orthogonal polynomials whose associated Jacobi matrix is a compact perturbation of the Jacobi matrix corresponding to the Chebyshev polynomials of the second kind. The above mentioned results on convergence of Turán determinants for the polynomials in the class $M(0,1)$ and their extension to the so-called shifted Turán (or Geronimo and Van Assche) determinants [8, Theorem 6 ] yield:

Proposition 1. Let the sequence of orthogonal polynomials $\left\{p_{n}\right\}$ be defined by the three-term recurrence relation

$$
\begin{gathered}
x p_{n}(x)=a_{n+1} p_{n+1}(x)+b_{n} p_{n}(x)+a_{n} p_{n-1}(x), \quad n \geq 0, \\
p_{-1}(x)=0, \quad p_{0}(x)=1,
\end{gathered}
$$

with real $b_{n}$ and positive $a_{n}$. Suppose that the recurrence coefficients satisfy $a_{n} \rightarrow$ $1 / 2$ and $b_{n} \rightarrow 0$ as $n$ diverges, and

$$
\sum_{k=0}^{\infty}\left(\left|b_{k+1}-b_{k}\right|+\left|a_{k+2}-a_{k+1}\right|\right)<\infty .
$$

Then the measure $\alpha$, with respect to which the $p_{n}$ are orthogonal, is absolutely continuous in $(-1,1), \alpha^{\prime}(x)>0$ for all $x \in(-1,1)$, and $\alpha^{\prime}$ is continuous in $(-1,1)$. 
Moreover,

$$
\lim _{n \rightarrow \infty} \delta_{n}(p ; x)=\frac{8}{\pi^{2}} \frac{1-x^{2}}{\left[\alpha^{\prime}(x)\right]^{2}}
$$

uniformly on the compact subsets of $(-1,1)$.

\section{REFERENCES}

1. R. Boas, Entire functions, Academic Press, New York, 1954. MR 16:914f

2. T. Craven and G. Csordas, Jensen polynomials and the Turán and Laguerre inequalities, Pacific J. Math. 136(1989), 241-260. MR 90a:26035

3. G. Csordas, T. S. Norfolk and R. S. Varga, The Riemann hypothesis and the Turán inequalities, Trans. Amer. Math. Soc. 296(1986), 521-541. MR 87i:11109

4. G. Csordas and R. S. Varga, Necessary and sufficient conditions and the Riemann hypothesis, Adv Appl. Math. 11(1990), 328-357. MR 91d:11107

5. J. Dombrowski, Spectral properties of phase operators, J. Math. Phys. 15(1974), 576-577. MR 48:13075

6. J. Dombrowski, Tridiagonal matrix representations of cyclic self-adjoint operators, I, II, Pacific J. Math. 114(1984), 325-334; 120(1985), 47-53. MR 87m:47046; MR 85h:47033

7. J. Dombrowski and P. Nevai, Orthogonal polynomials, measures and recurrence relations, SIAM J. Math. Anal. 17(1986), 752-759. MR 87g:42039

8. J. S. Geronimo and W. Van Assche, Approximating the weight function on several intervals, J.Approx.Theory 65(1991), 341-371. MR 93b:42037

9. G. Gasper, An inequality of Turán for Jacobi polynomials, Proc. Amer. Math. Soc. 32(1972), 435-439. MR 44:7013

10. S. Karlin and G. Szegő, On certain determinants whose elements are orthogonal polynomials, J. Analyse Math. 8(1960/61), 1-157; reprinted in: G. Szegő: collected papers, Vol. 3, (R.Askey, Ed.), Birkhäuser, Boston, 1982, pp. 605-773. MR 26:539

11. J. Mařík, On polynomials with all real zeros, Časopis Pěst. Mat. 89(1964), 5-9. MR 31:4782

12. A. Máté, P. Nevai and V. Totik, Asymptotics for orthogonal polynomials defined by a recurrence relation, Constr. Approx. 1(1985), 231-284. MR 89a:42035

13. G.V. Milovanović, D.S. Mitrinović and Th. M. Rassias, Topics in polynomials: extremal problems, inequalities, zeros, World Scientific, Singapore, 1994. MR 95m:30009

14. N. Obrechkoff, Zeros of polynomials, Publ. Bulg. Acad. Sci., Sofia, 1963.(in Bulgarian); German translation: Verteilung und Berchnung der Nullstellen Reeller Polynome, VEB Deutscher Verlag der Wissenschaften, Berlin, 1963. MR 29:1303

15. G. Pólya, Über die algebraisch-funktionentheoretischen Untersichungen von J. L. W. V. Jensen, Kgl. Danske Vid. Sel. Math.-Fys. Medd. 7 (1927), 3-33.

16. G. Pólya and J. Schur, Über zwei Arten von Faktorenfolgen in der Theorie der algebraischen Gleichungen, J. Reine Angew. Math. 144(1944), 89-113.

17. B. Riemann, Über die Anzahl der Primzahlen unter einen gegebenen Grösse, Monatsh. Der Berliner Akad. (1858/60), 671-680; also in: Gesammelte Mathematische Werke, 2nd edition, Teubner, Leipzig, 1982, No. VII, pp. 145-153.

18. G. Szegö, On an inequality of P. Turán concerning Legendre polynomials, Bull. Amer. Math. Soc. 54 (1948), 401-405; reprinted in: G. Szegő: Collected Papers, Vol. 3, (R.Askey, ed.), Birkhäuser, Boston, 1982, pp. 69-73. MR 9:429d; MR 84d:01082c

19. W. Van Assche and J. S. Geronimo, Asymptotics for orthogonal polynomials on and off the essential spectrum, J.Approx. Theory 55(1988), 220-231. MR 89m:33022

20. R. S. Varga, Scientific computation on mathematical problems and conjectures, Regional Conf. Ser. Appl. Math. Vol. 60, SIAM, Philadelphia, PA, 1990. MR 92b:65012

Departamento de Ciências de Computação e Estatística, ibilce, Universidade Estadual Paulista, 15054-000 SÃo José do Rio Preto, SP, Brazil

E-mail address: dimitrov@nimitz.dcce.ibilce.unesp.br 